Service social

\title{
La construction de soi chez les adolescents : Une histoire d'ouverture et de temps
}

\section{Marguerite Soulière}

Volume 59, numéro 1, 2013

URI : https://id.erudit.org/iderudit/1017483ar

DOI : https://doi.org/10.7202/1017483ar

Aller au sommaire du numéro

\section{Éditeur(s)}

École de service social de l’Université Laval

ISSN

1708-1734 (numérique)

Découvrir la revue

Citer cet article

Soulière, M. (2013). La construction de soi chez les adolescents : Une histoire d'ouverture et de temps. Service social, 59(1), 108-128.

https://doi.org/10.7202/1017483ar
Résumé de l'article

Cet article propose une réflexion sur la construction de soi des adolescents garçons. Après une mise en contexte sociopolitique et théorique de l'adolescence, il présente la démarche méthodologique d'une étude ayant pour objectif de comprendre l'adolescence des garçons de l'intérieur : une conjugaison de divers modes de collecte et d'analyse de données (création vidéos, interactions, entretiens de groupe et entrevues individuelles). Il rapporte que pour les garçons le processus de croissance qu'ils vivent entre 14 et 17 ans se représente en processus d'ouvertures : ouverture de soi, ouverture aux autres et ouverture au monde. L'adolescence se vit aussi dans une tension de double temporalité : un temps éminemment présent et un temps tourné vers l'avenir. Finalement, l'ethnographie présentée conduit à une discussion plus spécifique touchant l'identité masculine. Il est proposé d'ouvrir ce concept à l'idée de subjectivité plurielle, un processus individuel et collectif, toujours en mouvement et en interaction avec le contexte sociopolitique.
Ce document est protégé par la loi sur le droit d'auteur. L'utilisation des services d’Érudit (y compris la reproduction) est assujettie à sa politique d'utilisation que vous pouvez consulter en ligne.

https://apropos.erudit.org/fr/usagers/politique-dutilisation/ 


\title{
La construction de soi chez les adolescents : Une histoire d'ouverture et de temps
}

Marguerite Soulière

\section{RÉSUMÉ}

Cet article propose une réflexion sur la construction de soi des adolescents garçons. Après une mise en contexte sociopolitique et théorique de l'adolescence, il présente la démarche méthodologique d'une étude ayant pour objectif de comprendre l'adolescence des garçons de l'intérieur: une conjugaison de divers modes de collecte et d'analyse de données (création vidéos, interactions, entretiens de groupe et entrevues individuelles). II rapporte que pour les garçons le processus de croissance qu'ils vivent entre 14 et 17 ans se représente en processus d'ouvertures : ouverture de soi, ouverture aux autres et ouverture au monde. L'adolescence se vit aussi dans une tension de double temporalité : un temps éminemment présent et un temps tourné vers l'avenir. Finalement, l'ethnographie présentée conduit à une discussion plus spécifique touchant l'identité masculine. II est proposé d'ouvrir ce concept à l'idée de subjectivité plurielle, un processus individuel et collectif, toujours en mouvement et en interaction avec le contexte sociopolitique.

Mots-clés : Adolescence; anthropologie; analyse critique; analyse interprétative; garçons; masculinité; subjectivité plurielle; identité masculine.

\begin{abstract}
This article proposes a reflexion on the construction of how adolescent boys construct their self identity. After situating this in the sociopolitical and theoretical context of adolescence, it presents the methodology of a study aiming to understand from inside the adolescence of the boys: a mix of various modes of collection and analysis of data (the collective creation of videos, interactions, focus groups and individual interviews). It points out that for boys the process of growth they live between 14 and 17 years is represented in terms of openings: opening of oneself, opening to others and opening to the world. Adolescence is also lived as a tension of double temporality: a time eminently present and a time turned towards the future. Finally, the ethnography leads to a more specific discussion concerning the male identity. It is proposed to open this concept with the idea of plural subjectivity, an individual and collective process, always in movement and interacting with the sociopolitical context.
\end{abstract}

Key words: Adolescence; anthropology; critical analysis; interpretative analysis; boys; teenagers; masculinity; male identity; masculinities, plural subjectivity. 


\section{INTRODUCTION}

Dans le premier chapitre d'un ouvrage collectif sur la (re-)construction de «l'adolescent », Vadeboncoeur (2005) questionne la production des connaissances qui nous fait voir les adolescents comme « naturellement » fragiles et toxiques, en danger et dangereux. Concevant ces derniers dans une étape de développement entre-deux, une sorte de no man's land, un ensemble de discours dominants et populaires imposent une grammaire et un vocabulaire qui les définissent comme essentiellement victimes de leurs hormones, téméraires, influençables, dépendants de leurs pairs (Lesko, 1996). Cette vision se renforce et se «pathologise » avec une littérature savante et médiatique pointant les déviances réelles et potentielles des adolescents et sonnant constamment l'alarme à propos des multiples risques et dangers qui les (et nous) menacent (toxicomanie, conduites à risques, décrochage scolaire, violence, intimidation, dépression, suicide, gangs de rue, etc.). Les peurs que ces discours génèrent, qui plus d'une fois dégénèrent en panique morale, justifient de mettre en place des pratiques afin d'encadrer et de règlementer les comportements et la vie des adolescents (Kelly, 2003; 2007). Ce régime de vérité nous convainc de la nécessité de les garder sous un contrôle qui les préserve eux-mêmes, et les autres, des conséquences de leur choix irréfléchis et irrationnels (Vadeboncoeur, 2005).

Dans les faits, ces vérités inquiétantes qu'ont livrées les experts, qu'ont reprises les médias et dont se sont inspirées les institutions concernent une minorité de jeunes (Bouchard, 2011; Cousineau, 2011; Girard, 2011). II faut évidemment prendre au sérieux et s'occuper des difficultés que traversent des adolescents à un moment ou l'autre de leur parcours. Cependant, il faut être attentif aux effets de pouvoir (Foucault dans Piron, 1992) que crée le matraquage de peur et d'inquiétude, et à quel point cela influe sur notre manière de voir et d'agir avec les adolescents. C'est en effet ce maillage discursif (savant, médiatique et institutionnel) qui forge en bonne partie nos représentations de l'adolescence, et qui forcément donne le ton aux relations intergénérationnelles ${ }^{1}$. C'est aussi dans ce terreau culturel, social et politique que se construisent les adolescents aujourd'hui. Leur expérience de croissance et d'ouverture se fait à partir de ces repères, quelle que soit la position qu'ils adoptent face aux peurs et aux attentes réitérées de la société à leur égard.

L'intérêt anthropologique pour l'adolescence et la jeunesse d'une société donnée, qui se trouve à un moment donné de son histoire, vient du fait qu'elles sont des portes d'entrée privilégiées pour saisir les mécanismes de construction de soi, les appartenances et les héritages, les enjeux de changement et de continuité propres à cette collectivité située en ce lieu à ce moment précis. En effet, l'adolescence est une occasion de réitérer ou de reformuler les processus de socialisation et d'individualisation et leurs effets attendus, qui par ailleurs, concernent l'ensemble des individus, bien au-delà de cette période. Dit autrement, l'adolescence et la jeunesse sont de ces moments de transition que chaque société investit pour produire un ensemble de discours et de pratiques qui définissent les individus dont elle a besoin pour se maintenir en place et se développer (Lock, 1993).

1. Je ne peux m'empêcher de faire le lien avec la manière dont les étudiants du Québec ont été dépeints dans de nombreux médias et traités au printemps 2012 durant leur lutte contre la hausse des droits de scolarité. 
Ce présent texte s'intéresse à la construction de soi des adolescents garçons. II commence par situer celle-ci dans le contexte de notre époque et par rapport aux tendances qui dominent la théorisation de l'adolescence. Après la présentation de la méthodologie de l'étude dont sont issues les données, il enchaîne avec une vision de l'intérieur du processus de construction de soi pour des garçons entre 14 et 17 ans. Ensuite, une discussion plus spécifique pointe la transformation de l'identité masculine qui émerge de l'ethnographie et propose la notion de subjectivité plurielle. Et finalement, une brève conclusion donne le mot de la fin aux adolescents.

\section{Une mise en contexte}

Depuis les années 1960, dans les sociétés occidentales on voit se transformer de plus en plus définitivement le socle commun de valeurs, de pratiques et d'institutions cimentées dans des relations de pouvoir (entre les sexes, les générations, à l'intérieur d'une culture ou d'un groupe social). On assiste à une mutation des repères née d'une volonté affirmée individuellement et collectivement de rompre avec les appartenances généalogiques ou imposées par la tradition (Dubar, 2000). Le sujet apparaît dans l'espace social et avec lui les valeurs de liberté, d'égalité, de justice, de respect des individus et de leurs différences. II inspire nombre de discours, de pratiques et de politiques sociales ${ }^{2}$.

Depuis les années 1980, concomitant à cette rupture libératrice, un nouvel ordre social et politique s'impose et s'articule autour des discours de menace constante justifiant des pratiques de contrôle accru (Peretti Wattel, 2000) ${ }^{3}$. Étroitement lié à la pensée unique, pragmatique de l'économique, cette logique du risque et d'évitement du pire (Beck, 2001) s'immisce dans toutes les sphères de la vie et façonne nos représentations de soi, de l'Autre et du monde. C'est ainsi que ce nouveau paradigme de la menace et du contrôle assujettit par la peur les États, les institutions, les entreprises et les individus aux dogmes de la marchandisation, de la compétitivité et de la croissance (Petrella, 2007). On se réfère alors aux personnes (et à leurs comportements) comme à des entités séparées et homogènes, responsables isolément de leurs défaites et leurs réussites. C'est dans cette (dés-) articulation historique et épistémologique paradoxale de subjectivation émancipatrice et d'assujettissement que se pose aujourd'hui la question des identités et, plus spécifiquement ici, celle de la construction de soi des adolescents. Dans cette époque de paradoxe et d'incertitude, on se demande quels sont les pôles d'appui que mettent en place, tant les adultes pour transmettre le monde déjà là (Arendt, 1975), que les adolescents pour y donner sens et s'y faire une place.

2. Ces transformations se préparent depuis la fin du XVIII ${ }^{e}$ siècle, avec la Révolution française; à la fin du $X I X^{e}$ siècle Nietzsche prédisait la venue de «l'individu souverain, qui n'est semblable qu'à lui-même », et au milieu du $X X^{\mathrm{e}}$ il « est désormais une forme commune de vie » (Ehrenberg, 2000, p. 14).

3. Je réfère ici à la société du risque qui, selon U. Beck, est une condition mondialement partagée des sociétés postindustrielles. La transition est survenue lorsqu'à la suite à d'accidents industriels aux conséquences catastrophiques imprévisibles et incontrôlables (Bhopal, Tchernobyl, etc.), la perception de dangers et de menaces s'est mise à guider les représentations et les pratiques de ces sociétés. 


\section{Théories de l'adolescence et construction de soi}

La plupart des auteurs qui traitent de l'adolescence font remonter ses premières théorisations scientifiques à Stanley Hall (1844-1924), surnommé «le père de l'adolescence » (Vadeboncoeur, 2005). Formé auprès d'évolutionnistes, Hall fut un des principaux précurseurs de la psychologie développementale. Il compare les étapes du développement cognitif et psychologique des individus aux stades de l'évolution des groupes humains, tels que modélisés par les premiers anthropologues ${ }^{4}$. Produits $d u X I X^{e}$ siècle, en pleine industrialisation et urbanisation des sociétés nord-américaines, ces discours évolutionnistes s'inspiraient de l'essor du capitalisme et ses principes de croissance et de progrès pour théoriser le développement de l'humanité (Lesko, 1996).

Débordant le cadre de la psychologie, c'est sur ce modèle que s'est construite et renforcée l'idée que les enfants et les jeunes gens sont naturellement des êtres inachevés, en stade de progression vers le but à atteindre: devenir un adulte. Ainsi, les adolescents, situés entre l'enfance et la vie adulte, sont considérés comme fragilisés par leur biologie transitoire et leur immaturité affective et cognitive. C'est sur ces postulats théoriques que reposent aujourd'hui nombre de cadres de référence pour analyser l'adolescence et mettre en place des programmes en vue de protéger les adolescents eux-mêmes et la société des dérives potentielles de leur immaturité.

Pourtant des écrits majeurs de la sociologie française (Galland, Singly, Le Breton) notamment, réfutent cette prédominance de la biologie et de la psychologie dans la compréhension de l'adolescence. Pour ces sociologues, ce réductionnisme à un processus universel de développement homogénéise à tort l'expérience adolescente et ne tient pas compte de la grande variabilité des contextes socioculturels, de l'époque et des conditions de vie, des caractéristiques et des trajectoires personnelles. Insistant surtout sur la construction sociale de l'adolescence toujours contingente à la société (et l'époque) qui la produit, ces auteurs se positionnent néanmoins de manière distincte dans ce qu'ils rapportent de l'adolescence aujourd'hui. Comme le fait remarquer Duret (2008), deux tendances se dessinent : une première qui trace le portrait d'adolescents qui vont bien, la majorité selon les statistiques, et la deuxième qui met l'accent sur les parcours de ceux qui souffrent, une minorité d'entre eux, leurs difficultés et les risques auxquels ils s'exposent. II va sans dire que, suivant la tendance à laquelle on se rattache, la lecture de la construction de soi des adolescents et de la société dans laquelle ils vivent, s'en trouve nuancée.

François de Singly, chef de file de la première tendance, met en lumière le processus d'individualisation des «adonaissants », dès leur entrée au secondaire. Comme il le fait dans de nombreux ouvrages, Singly explique les effets des transformations sociales qui ont libéré les sujets de l'obligation de conformité à une identité liée à l'appartenance à un sexe, une famille, une religion. Pour lui, l'individualisation (ou l'autonomie) :

4. Parmi ceux-ci, Lewis Morgan, dans son ouvrage Ancient Society avance une théorie de l'évolution des sociétés et propose une typologie (sauvages, barbares et civilisées) pour catégoriser (et hiérarchiser) les sociétés en fonction de leur stade de développement technologique et économique (Paul Bohannan et Mark Glazer (dir.), (1988), « Lewis Henry Morgan » dans High Points in Anthropology, New York, McGraw-Hill, p. 29-60.). 
C'est le droit pour tout individu de ne pas être défini seulement par une place dans l'ordre des générations, des sexes, ou encore dans telle ou telle institution. (....) Chacun est défini comme auparavant par sa place, mais il peut également être considéré en tant qu'individu à part entière, en tant que personne. Le processus d'individualisation exprime un refus devant la réduction identitaire » (Singly, 2006, p. 12).

Alors, ce qui l'intéresse, c'est la manière dont les jeunes bricolent leur processus d'individualisation ou de construction de soi qui, dans nos sociétés hypermodernes, est devenu moteur de la socialisation (Kaufman, 2007). Contrairement aux idées reçues sur la «crise d'adolescence », les recherches de Singly auprès des jeunes l'amènent à penser que la naissance du «je » adolescent ne s'opère pas au moyen d'une rupture fracassante avec le milieu familial, mais dans un processus d'agencement compatible (et complémentaire) entre le « nous » des amis et le « nous » familial. C'est à mesure que le « je » prend du coffre (et c'est dans la lutte contre le réductionnisme identitaire à l'un ou l'autre qu'il le fait) qu'un adolescent fait ses choix et se construit comme personne. Ce que Singly explique ainsi :

l'histoire de l'individualisme en Occident repose sur le principe de la séparation et sur la non-confusion entre les appartenances, les positions et l'identité personnelle. (...) La distinction entre les deux dimensions identitaires n'implique pas que les appartenances, les positions, les statuts n'ont aucun sens pour l'individu qui ne serait heureux que dépouillé, que désaffilié. (...) l'individu dispose d'un pouvoir sur lui-même qui l'autorise à juger ce qui est pertinent pour lui (Singly, 2005, p. 359).

Singly soutient que la (recon)naissance du sujet dans les institutions et l'espace social en général lui offre la possibilité de se construire librement et consciemment en conjuguant de multiples appartenances, parfois contradictoires. En ce sens, être autonome ne veut pas toujours dire être indépendant (de ses parents, des ses amis), «mais de considérer l'importance de ce lien, même si parfois cela demande de faire des concessions sur sa liberté personnelle » (Singly, 2006).

La société des individus à laquelle fait référence François de Singly, et dans laquelle se construisent les adolescents d'aujourd'hui, n'est pas dépourvue de liens, de cadre ou de valeurs, au contraire, ils se bâtissent individuellement et collectivement sous les principes d'égalité et de liberté (Singly, 2006). Encore faut-il que les adolescents soient entourés et soutenus pour le faire (Duret, 2008).

Prenant appui sur un autre cadre d'analyse, David Le Breton, chef de file de la deuxième tendance, est auteur de plusieurs ouvrages sur l'adolescence et ses souffrances (2003; 2007). II se penche plutôt sur les adolescents «écorché vifs », ceux qui errent, seuls, en quête de sens. Le Breton met en rapport l'adolescence «à risque » et les profondes transformations sociétales qui laissent sans repères les plus fragiles d'entre eux. Regroupant les conduites à risque sous quatre grandes catégories anthropologiques (ordalie, sacrifice, effacement de soi et affrontement), il les interprète avant tout comme des cris de détresse et des appels à l'aide et 
aussi, pour certains, des moyens de se mettre au monde, de naître en quelque sorte à travers la force psychique qu'appelle la blessure infligée ou le côtoiement de la mort (Le Breton, 2003).

Selon lui, les pressions de performance qui pèsent sur les individus conjuguées à la rupture des familles, la démission des parents et leur refus de vieillir, la perte d'autorité, le chacun pour soi et l'effritement des valeurs communes font en sorte qu'il est difficile pour les adolescents de se situer et de trouver leur place dans les sociétés hypermodernes. II rejoint d'autres sociologues de la famille inquiets des dérives de la jeunesse actuelle, tout comme de celle des institutions qui avaient fonction de balises et d'encadrement dans les sociétés traditionnelles. Ces sociologues établissent des corrélations entre les maux sociaux, auxquels les adolescents «à risque » participent, et une perte de repères et d'assises solides que les sociétés occidentales n'arrivent plus à offrir depuis la fin des années 1960 (Dagenais, 2000; Le Breton, 2003).

C'est dans une tension paradoxale indéniable que se pense et s'appréhende l'adolescence aujourd'hui. La chose n'est sans doute pas étrangère à la « pathologisation » des conduites des adolescents. Les enjeux majeurs des sociétés occidentales se sont plus d'une fois cristallisés sur les discours et les pratiques entourant l'adolescence: les sociétés en transformation s'inquiètent des capacités des générations montantes de faire face aux défis de l'avenir, surtout lorsque ceux-ci dépassent les générations adultes (Passerini, 1994). En observant sans comprendre les nouvelles conduites des jeunes, les institutions cherchent à éviter les dérives, à mettre en place des dispositifs de contrôle pour éviter leur perte (et la nôtre). C'est dans ce rapport au monde particulier et situé que prennent source les discours alarmistes et inquiets qui font écran à ce qui se vit à l'intérieur même de l'adolescence. Les représentations pathologisantes qui en découlent maintiennent dans le brouillard les repères émancipateurs dont héritent les générations montantes.

C'est dans cet environnement sociopolitique, cadrés qu'ils sont dans des programmes et des politiques, que les adolescents grandissent et se fabriquent comme personne et comme génération. Pour mieux comprendre comment ils appréhendent ou s'approprient cet environnement paradoxal de subjectivation émancipatrice et d'assujettissement, je propose d'aborder les adolescents autrement que dans leurs travers, leurs manques et leurs problèmes. Je propose une plongée à l'intérieur de l'adolescence en donnant à des garçons entre 14 et 17 ans un espace d'expression de leur expérience élaborée à partir de leur rapport à soi, aux autres, au temps et à l'espace.

\section{UNE PLONGÉE DANS L'ADOLESCENCE : DÉMARCHE MÉTHODOLOGIQUE}

L'ethnographie présentée ici a été réalisée en Estrie au Québec en 2004-2005. L'échantillon est composé de 17 garçons québécois, d'origine canadienne-française, âgés de 14 à 17 ans, répartis en trois groupes. La répartition avait été définie lors d'une étape préliminaire, lors de laquelle des adolescents avaient identifié les catégories conceptuelles qui organisent leur monde: des lieux d'appartenance, des liens d'attachement et des activités pratiquées (partagées pour la plupart). C'est en croisant ces trois principes organisateurs de leur viemonde (lieu, lien, activité) et en privilégiant pour chacun la composante la plus forte, comme les 
adolescents eux-mêmes la rapportent, que les trois groupes furent constitués : un premier sur la base de la fréquentation d'un lieu, l'école (8 ados); un deuxième sur la base de liens, une gang de meilleurs amis (4) et enfin un troisième sur la base des activités, l'activité-passion de faire des films (5). Le groupe scolaire a été formé, sur une base volontaire, dans une école secondaire de l'Estrie, connue pour des actions menées pour répondre aux difficultés spécifiques des garçons. Le groupe d'amis a été approché par recrutement direct dans le quartier où j'habitais à Sherbrooke. Et enfin les passionnés de vidéo ont été rassemblés grâce à des annonces publiques dans des soirées de présentation de vidéos amateurs et dans des écoles secondaires de Sherbrooke qui offraient une activité-création de courts métrages en parascolaire.

Bien que l'échantillon ait été formé à partir de catégories émiques ${ }^{5}$, des données sociodémographiques recueillies lors des entrevues individuelles, témoignent de la diversité des provenances des participants.

\begin{tabular}{|l|l|}
\hline \multicolumn{2}{|l|}{$\begin{array}{l}\text { Revenu de la résidence } \\
\text { principale }\end{array}$} \\
\hline $10000-24999$ & $2 / 14$ \\
\hline $25000-32999$ & $2 / 14$ \\
\hline $33000-45999$ & $5 / 14$ \\
\hline $46000-59999$ & $2 / 14$ \\
\hline $60000-75999$ & $0 / 14$ \\
\hline 76000 et plus & $3 / 14$ \\
\hline
\end{tabular}

\begin{tabular}{|l|l|}
\hline \multicolumn{2}{|l|}{$\begin{array}{l}\text { Cohabitation avec les } \\
\text { parents/ \% temps }\end{array}$} \\
\hline $\begin{array}{l}\text { Père et mère } \\
100 \%\end{array}$ & $5 / 14$ \\
\hline $\begin{array}{l}\text { Père } 50 \% \text { mère } \\
50 \%\end{array}$ & $1 / 14$ \\
\hline $\begin{array}{l}\text { Père (70\% et } \\
\text { plus) }\end{array}$ & $2 / 14$ \\
\hline $\begin{array}{l}\text { Mère (86\% et } \\
\text { plus) }\end{array}$ & $2 / 14$ \\
\hline $\begin{array}{l}\text { Mère et conjoint } \\
\text { (90\% et plus) }\end{array}$ & $3 / 14$ \\
\hline Famille d'accueil & $1 / 14$ \\
\hline
\end{tabular}

\begin{tabular}{|l|c|}
\hline \multicolumn{2}{|l|}{ Milieu géo-économique } \\
\hline $\begin{array}{l}\text { Urbain (plus de } \\
100000 \mathrm{~h})\end{array}$ & $4 / 14$ \\
\hline $\begin{array}{l}\text { Semi-urbain (moins } \\
\text { de } 100 \text { 000) }\end{array}$ & $9 / 14$ \\
\hline Rural & $1 / 14$ \\
\hline
\end{tabular}

5. Les catégories émiques sont issues de la logique interne propre à un individu ou à un groupe, en interface des catégories étiques qui renvoient, elles, à la perspective et aux analyses du chercheur. 
Chacun des trois groupes de participants a d'abord été invité à créer (de la première tempête d'idées au montage final) une vidéo en 24 heures (huit semaines à raison d'une séance de trois heures par semaine pour les deux premiers groupes et trois journées de huit heures pour le dernier) sur le thème et dans le style de leur choix. Cette première activité de collecte de données a été complétée par des entretiens de groupe et des entrevues individuelles ${ }^{6}$. Dans le souci de recourir à une méthodologie adaptée aux sujets (Nyesito, 2000), cette pluralité de modes d'expression a ouvert des possibilités de conjuguer la création individuelle et collective, les interactions, la réflexivité, l'imagination et l'intuition (Bruner, 1986).

Les outils de collecte des données (animation des créations, guide d'entretien de groupe et guide d'entrevues individuelles) ont été préparés suivant les différentes composantes (identité, altérité, temporalité et spatialité) du cadre conceptuel de la recherche : le Corps adolescent. Ce modèle que j'ai élaboré pour appréhender l'adolescence est inspiré du Mindful Body ${ }^{7}$, un cadre de référence proposé comme préalable aux travaux en anthropologie médicale. II propose de mettre en dialogue les dimensions individuelle, sociale et politique, qui sont toujours en cause dans l'étude des phénomènes humains et sociaux. C'est dans cette perspective que le corps adolescent a été considéré comme une scène sur laquelle une génération crée, à même sa culture, l'immédiat, son temps et son espace. Le Corps adolescent évoque à la fois la démarche de recherche et la complexité inhérente au phénomène de l'adolescence.

\section{CADRE CDNCEPTUEL \\ Corps adalescent}

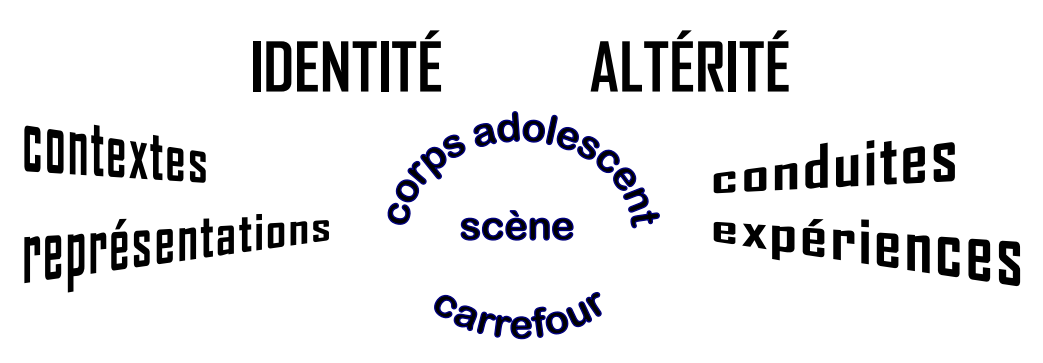

TEMPQRALITÉ SPATIALITÉ

Inspiré de Mindful Bady Margaret Lock et Nancy Scheper-Hughes

6. Pour des raisons personnelles à chacun, trois d'entre eux n'ont pas participé à l'entrevue individuelle.

7. Élaboré par deux anthropologues, Margaret Lock et Nancy Scheper-Hughes, en vue de poser des repères épistémologiques pour la recherche en anthropologie médicale. Pour une description détaillée du modèle, voir la référence en bibliographie. 
Les analyses en profondeur ont été faites pour chacune des trois sources de données: créations de vidéos, entretiens de groupe, entrevues individuelles. Les créations de vidéos ont été filmées, de manière à prendre en compte tant le processus de création que les fictions réalisées. Les entretiens de groupe et les entrevues individuelles ont été enregistrés et retranscrits intégralement. Tout au long du processus d'analyse (phénoménologique et herméneutique), une lecture en écho des différentes sources - les créations vidéo (l'histoire, les thèmes, les personnages, la construction dramatique, etc.); les processus de création (le cheminement des idées, les interactions); les entretiens de groupe (les explications des garçons et les discussions thématiques sur leur film et ceux des autres groupes, la réflexion sur leur expérience collective; les entrevues individuelles (les biographies personnelles, la réflexivité, les saillances, les silences, les structures narratives, la sémiologie populaire) - les a renforcées mutuellement et a donné un large éclairage sur « le dedans » de l'adolescence des garçons.

\section{SE CONSTRUIRE À L'ADOLESCENCE : LE PROCESSUS VU DE L'INTÉRIEUR}

La trame de fond qui se dessine à travers les récits et les films des adolescents, qui racontent ce qu'ils sont en train de vivre, renvoie à l'idée d'ouverture. Il est question d'ouverture de soi qui fait réaliser la sortie de l'enfance, d'ouverture aux autres, qui ponctue et imprègne de part en part la vie quotidienne, et d'ouverture au monde, à la fois omniprésente et reportée à la fin d'une étape consacrée, disent-ils, à se connaître soi-même.

La structure dramatique et le langage filmique d'une des réalisations des garçons illustrent très bien ce processus de triple ouverture. On suit le parcours d'un jeune adolescent dans une école secondaire qui au début est rejeté par ses camarades. Ensuite, il trouve le moyen de se faire accepter et, finalement, le jeune héros meurt, tout de suite après son entrée dans le monde scénarisée par un don de sang pour faire son devoir de citoyen. Malheureusement, une infection au sang contaminé lui coûtera la vie... Le film avait pourtant une tout autre intention : un message humoristique sur les méfaits de la cigarette...

Ce processus d'ouverture se joue par ailleurs sur une double temporalité - l'avenir à préparer et le présent à célébrer - qui explique bien les tensions qui dynamisent la construction de soi chez les garçons. Les garçons ont incorporé la représentation dominante de l'adolescence comme une étape de transition, circonscrite dans le temps: elle a un début, une durée limitée et une fin. Son temps compté l'entoure d'une connotation d'urgence. S'ils ont compris qu'il est impératif de préparer son avenir et de faire des choix en conséquence (travailler à l'école, bien réussir, adopter de bonnes habitudes de vie, etc.), ils retiennent par ailleurs qu'il faut profiter de ce «temps précieux qui ne reviendra pas » pour s'amuser et faire des expériences. Pour la plupart des garçons, il s'agit en effet d'un temps éminemment présent, qui prend tout son sens dans l'urgence d'être et de vivre ici et maintenant. 
Fêter - faire le party -, moi, ça me comble ben raide. Quand je peux lâcher mon fou, niaiser avec mes chums... pis faire le cave dans nos films, ça, y a rien que j'aime plus que ça. C'est vraiment ça que je préfère et je veux vraiment finir mon adolescence et dire : - Yes ! J'ai eu du fun.

Benoît, 16 ans

C'est le temps.... Pis on cherche juste à profiter un petit peu du temps qu'on a...

Grégory, 16 ans

D'ailleurs, ceux qui ne prennent pas le temps de sortir et de s'amuser ne sont pas toujours bien vus. D'après ce que les garçons m'ont raconté, plusieurs voient dans le fait de se consacrer à ses études au point de ne pas accorder de temps à ses amis le signe de priorités mal placées, d'ambitions matérielles et professionnelles démesurées.

\section{L'ouverture de soi}

L'expérience d'ouverture de soi des garçons prend diverses formes, tout en se rattachant à des impressions d'élargissement, de déploiement et d'affirmation. Ce qui change dans le cours de l'existence et fait prendre conscience d'être en train de grandir se manifeste d'abord dans le rapport à soi. Pour certains, c'est comment ils se sentent et comment ils s'expriment en présence des autres. Ce processus d'ouverture est plus marqué au début du secondaire.

Ce que je trouve plus facile maintenant c'est de m'exprimer. Je pense que je suis content parce qu'avant j'étais gêné... pis j'avais peur des réactions des gens... Sauf qu'à c't'heure, je me dis je vais y dire ce que je pense.

Loïc, 16 ans

Il se poursuit tout au long de l'adolescence (et du secondaire, le deux se superposant dans les récits) :

Je suis au secondaire... c'est pas mal ça... Je suis encore entre le monde des adultes et le monde des enfants.

Loïc, 16 ans

Pour d'autres, c'est la découverte de nouveaux talents ou intérêts grâce à de nouvelles activités pratiquées (des jeux vidéo aux activités sportives, artistiques ou humanitaires).

Et là, c'est vraiment un moment où je m'intéresse à tout, pis... je suis toujours en train de regarder les comportements, me poser des questions, je pense beaucoup. [...] J'appellerais ça la période qui m'a allumé sur ce qui m'entoure... qui m'a plus allumé que juste vivre... [...] La période où j'apprends à connaître.

Maxance, 16 ans 
Pour certains, l'ouverture de soi passe par des expériences inédites.

La drogue, j'ai pris ça comme une expérience, j'ai fait le tour, je me suis dit, je me dis encore d'ailleurs, ce qui est nouveau, tant que tu n'abuses pas, ça peut juste t'enseigner autre chose pis moins te fermer les yeux et être plus ouvert sur ce qui se passe autour, moins porter de jugement.

Laurent, 22 ans $^{8}$

Ce nouveau rapport à soi s'exprime aussi (étonnamment, par rapport à ce qu'on dit de cet âge) par le besoin de solitude. La recherche de solitude est motivée différemment suivant les besoins de chacun : se retrouver seul avec soi-même, vivre des expériences de dépassement (en sport solitaire) ou, simplement, faire le vide, créer ou réfléchir.

Ça, c'est mon côté solitaire, parce qu'il est quand même présent... J'ai beau être extraverti mais... j'ai besoin de me retrouver souvent et de savoir ce que je veux parce que des fois je me perds... y a tellement de monde influent que je sais plus où me situer là-dedans, dans le monde.

Olivier, 16 ans

L'ouverture de soi apparaît finalement de manière tangible dans l'importance que prennent les choix personnels en matière de musique et de vêtements, qui chez plusieurs vont de pair. Au début de l'adolescence, cette urgence établit une nécessaire frontière symbolique entre le monde de l'enfance, dans lequel maman-papa décident des vêtements à porter, et le monde de changement qu'ils commencent à découvrir. S'il y a là une dimension uniforme propre à chaque époque (un éventail restreint de styles ${ }^{9}$ ), du point de vue de plusieurs, leur choix d'un style particulier vient de ce que celui-ci leur convient à eux.

Avant, quand j'étais plus jeune, comme Secondaire I et II, je voulais m'identifier à un groupe... le groupe plus hip-hop et rap - [...] ce style-là les culottes plus baissées... les gros gilets plus amples... la calotte de côté... pas la calotte de côté, ça j'aimais pas ça... Mais j'avais ça... je me sentais bien là-dedans.

Pierre-Luc, 15 ans

Plus on avance dans les années du secondaire, moins strictes sont les règles vestimentaires, moins vitales sont les griffes. Certains ont le goût de se démarquer par un habillement original tandis que d'autres y accordent moins d'importance (à l'intérieur de certaines normes quand même). Dans le film, quand le personnage principal était un « rejet » à

8. J'ai intégré ici un extrait d'entretien de groupe mené dans le cadre de cette même recherche, cette fois auprès de personnes à proximité des adolescents (parents, professeurs, intervenants et, ici, un groupe de jeunes hommes âgés de 21 à 25 ans, quelques années après la fin de leur secondaire). II n'aurait pas été possible d'avoir ce témoignage des adolescents au moment où je les rencontrais. La politique de « tolérance zéro » dans les écoles secondaires faisait en sorte qu'on ne peut pas parler de consommation de « drogue ». C'est d'ailleurs ce que m'ont confié les participants quelques années plus tard, quand je les ai regroupés pour valider mes analyses à la fin de ma thèse.

9. Rappeurs, Punks, Gothiques, Skas, Alternes, etc. 
l'école, il était habillé dans un style visiblement démodé. Quand il réussit à se faire accepter de ses pairs, il réapparaît avec des vêtements cools. L'espace de liberté que chacun arrive à se donner au fil des années se traduit aussi dans une souplesse et une ouverture aux styles des autres.

\section{L'ouverture aux autres}

L'école secondaire est le lieu central, pivot dans la vie des adolescents ${ }^{10}$. On y entre, la plupart du temps avec sa gang du primaire ou de son quartier. Avec le temps, tranquillement ces premiers amis changent parce qu'on se découvre de nouvelles affinités, plus personnelles, avec d'autres. Ces autres avec qui on apprend, on partage des intérêts, des manières de voir, des activités (des passions souvent) communes.

L'ouverture aux autres veut aussi dire apprendre à connaître et à apprécier une grande diversité de personnes. Si les premières années du secondaire sont sous le signe de la division en clans identitaires, la fin est marquée par la reconnaissance de la communauté qu'en est venue à créer la cohorte, principalement durant la dernière année.

En plus on dirait que cette année, avec du monde que tu pensais jamais avoir des liens, ça s'est formé parce que, quand t'es en Secondaire $V$, ce n'est pas comme avant où c'était gang par gang. Mais là, asteure, tout le monde se parle et c'est le fun.

Louis, 16 ans

Le bal et les après-bals ont dans une large part une fonction rituelle de célébrer et de clore l'expérience commune de traversée du secondaire.

C'est comme la dernière grosse fête tout le monde ensemble... on se voit toute... pis c'était vraiment symbolique si je peux dire. On se dit : «Bye! » Surtout avec le monde qui habite loin, qu'on ne voit pas souvent... « notre » gang, pas « la » gang... C'est notre école.

Ludovic, 17 ans

L'ouverture aux autres c'est aussi entretenir une multitude de relations et faire une différence entre les amis Facebook, les amis d'école ou d'enfance et les vrais amis. Ces catégories ne sont pas exclusives, et même si les amis Facebook ont par définition moins d'importance, on retrouve sur Facebook les amis de l'école et tous ses vrais amis. Tous les garçons rencontrés s'entendent sur les valeurs qui fondent de vraies amitiés : le respect (« on se prend comme on est »); l'authenticité (« on se dit les vraies affaires »); la confiance (« on peut me dire quelque chose, je ne le dirai à personne »); et l'amour («je considère que mes amis, là, c'est de l'amour »).

10. Même pour ceux qui quittent l'école, elle reste un lieu fréquenté régulièrement : pour voir les amis évidemment. 
C'est dans l'intimité des relations ${ }^{11}$ où « on se dit tout », qui sont là pour toujours, qu'on se connaît, qu'on se bâtit et que, réciproquement, on s'aide à grandir. L'intimité prend diverses formes. C'est une base de sécurité, un cadre rassurant de transformation :

Moi, je les perçois comme des personnes que j'aime... que j'aime beaucoup parce que, justement, en leur parlant de ma vision, je la comprends mieux. Et ils me parlent de leur vision, ils m'apportent des choses et, en discutant ensemble, on se comprend mieux. C'est comme une évolution qui s'est faite rapidement sans qu'on le veuille... sans qu'on le décide $[. .$.$] .$

Maxance, 16 ans

C'est une communion dans des expériences inédites :

On va dans la nature, on se perd complètement et on reste là pendant des heures à rien faire. Et on réussit à aller toucher quelque chose qui est incomprenable.

Maxance, 16 ans

L'intimité c'est aussi de se remettre à l'ordre entre eux quand ils jugent qu'il y a consommation excessive d'alcool ou de drogue, par exemple, ou encore, c'est de tenter de dissuader un des leurs qui est sur le point d'arrêter les études.

Glorifiant le mode de l'humour, les garçons ensemble se font avant tout le plaisir (et le devoir) de s'amuser, de rire, de déconner pendant que c'est le temps. C'est maintenant, disentils, qu'il faut faire le plus d'expériences possible et dépasser ses limites pour les connaître. De plus, nombre de «conduites à risque » sont réinterprétées par les garçons comme des manières de se souder entre eux, de marquer ce temps mémorable par des récits d'exploits qui les fédèrent, les mettent au monde en dehors des repères de l'enfance et de la famille ${ }^{12}$.

On se payait des shooters, 130 shooters à trois, j'ai une pyramide de shooters plastifiée à la maison

Bernard, 22 ans $^{13}$

Ce présent-à-tout-prix parle aussi de ce double horizon sur lequel se projettent et agissent nombre de garçons de cet âge. À fond dans le plaisir immédiat, et ayant derrière la tête cette nécessité de vivre tout cela maintenant, parce que les responsabilités qui s'en viennent seront d'autant plus faciles à assumer qu'on aura profité des expériences d'éclatement, de défis et de conquêtes. À l'interface de cette notion de plaisir, on pourrait presque dire « prescription », il y a

11. Pour une théorisation de l'intimité au masculin, lire G. Dulac (2003), "Masculinité et intimité », Sociologie et sociétés, vol. 35, no 2, p. 9-34.

12. Pour en connaître plus sur une perspective interprétative de la prise de risque : Cynthia Lightfoot (1997), The Culture of Adolescent Risk-Taking, New York, Guilford Press.

13. Il s'agit d'un extrait d'entretien de groupe mené dans le cadre de cette même recherche, cette fois auprès de personnes à proximité des adolescents (parents, professeurs, intervenants, et ici, un groupe de jeunes hommes entre 21 et 25 ans, quelques années après la fin de leur secondaire. 
celle de responsabilité. Comme plusieurs envisagent de s'engager amoureusement et d'avoir des enfants (plus tard), c'est maintenant, pendant qu'ils sont seuls, qu'ils peuvent faire leurs expériences, sans déranger ni faire souffrir quelqu'un. Cette vision de leur adolescence est souvent héritée des hommes significatifs de leur entourage.

En effet, l'ouverture aux autres dans le processus de construction de soi se manifeste aussi dans le rapport aux grands, aux adultes qui les entourent. Le plus significatif est souvent le père, il y a aussi le grand frère et les adultes qui leur accordent du temps (voisins, amis, professeurs, entraîneur ou animateurs), qui s'intéressent à eux ou avec qui ils partagent une passion. Même si les garçons rapportent que leur mère est la personne de qui ils sont le plus proches (28\%, contre $21 \%$ pour le père), la grande majorité $(79 \%)$ considèrent leur père comme l'adulte masculin le plus significatif, et ce, en dépit du fait que $64 \%$ d'entre eux aient vécu la séparation de leurs parents durant l'enfance. Ce qu'ils retiennent de plus puissant chez leur père (et pour certains, leur grand-père), ce sont, suivant les participants, des valeurs de courage d'être soi-même, du travail bien fait, de persévérance, d'engagement et de responsabilités.

Mon père m'a toujours dit que, quand tu travailles, tu donnes tout ce que t'as... tu fais le mieux.

Loïc, 16 ans.

\section{L'ouverture au monde}

Elle est unanime, cette ouverture au monde, chez les répondants de cette étude : ambivalente, à la fois rassurante et inquiétante. Elle apparaît dans les films, dans les activités de création et les récits qui racontent ce qu'on rêve de faire plus tard et ce qu'on ne veut surtout pas.

L'ouverture au monde pour les garçons se fait sur deux horizons : le premier est celui de la réalisation personnelle (un travail et une famille) et de l'indépendance économique qui consacrera leur place dans le monde; le deuxième horizon est celui de la conscience des enjeux économiques, environnementaux et politiques. Ces deux aspects de la vie qui les attend sont racontés en parallèle. À un niveau personnel, la réalisation de leurs rêves d'avenir ne semble pas un problème dans la mesure où ils croient qu'en travaillant ils vont y arriver.

Ils se disent conscients du contingentement des programmes de formation, surtout les plus gratifiés socialement et économiquement. Pour cette raison, ceux qui se destinent à de tels programmes essayent de conjuguer bons résultats scolaires et plaisir. Par ailleurs, ceux qui ne savent pas trop où se diriger (le cas de la grande majorité) aperçoivent un avenir aux portes ouvertes, aux multiples possibilités de formations et d'emplois : il ne suffira qu'à s'y engager quand on aura fait un choix. 
En revanche, pour ce qui est de la société, du monde dans lequel ils auront à vivre comme génération, l'avenir paraît plutôt sombre, voire désespérant.

La race humaine va disparaître et y va y avoir beaucoup d'affaires qui vont partir un moment donné... Ça va prendre du temps, mais c'est sûr qu'on est en train de péter.

Benoit, 16 ans

Je trouve que de la façon que c'est géré. Tant que ce ne sera pas épuisé, genre, on ne changera pas. Tant que ça marche et qu'on fait de l'argent, on ne changera pas.

Jeremy, 16 ans

Ils reconnaissent vivre dans l'abondance, à un endroit privilégié de la planète. Par contre, à l'interface de la richesse de leur pays se trouve la pression de consommation, le piège de l'endettement : « le tombeau des rêves et des passions ». D'ailleurs, une des vidéos réalisées a pour titre Mégasurface. On y voit un jeune homme égaré pendant trois jours dans un supermarché, magiquement transformé en «mégasurface », alors qu'il s'y était rendu pour acheter du pain en vitesse pour son déjeuner. Tout ce temps, il tourne en rond et se fait constamment harceler par des vendeurs postés à des stands de nouveaux produits. Pour finir, il retrouve son chemin, et rentre chez lui pour découvrir en arrivant qu'il lui restait du pain dans l'armoire. Il en meurt sur le coup. Et cette phrase d'apparaître à l'écran : « Les mégasurfaces ne tuent pas que les petits commerces. »

Plusieurs ont peur d'être forcés de faire un travail qu'ils n'aiment pas pour payer une hypothèque et des cartes de crédit. Ils se préoccupent aussi de leur endettement collectif : qui va payer pour les déficits accumulés ? Ils prévoient payer beaucoup plus d'impôt que leurs parents et plusieurs déplorent l'utilisation que fait l'État des fonds publics, dans l'armée par exemple.

Très rarement le pouvoir politique leur paraît une solution. Ils ne conçoivent pas le gouvernement comme pouvant changer le cours des choses. Il y a la responsabilité individuelle, comme celle de protéger l'environnement (chacun pose des gestes de protection : récupérer, mettre ses déchets dans les poubelles, etc.). Mais, à grande échelle, collectivement, plusieurs se sentent impuissants: ils perçoivent leur société sous le joug des intérêts financiers que favorisent les multinationales et les États ${ }^{14}$. Il n'est donc pas surprenant de les entendre déplorer les motivations d'enrichissement personnel des politiciens. Ce qui, en fait, leur apparaît comme le reflet de la majorité individualiste qui ne se préoccupe que de sa petite affaire et veille, chacun pour soi, à tirer son épingle du jeu. Pourtant certains, les plus optimistes, croient que lorsque les vieux seront délogés, leur génération, beaucoup plus consciente des enjeux planétaires, en matière d'environnement surtout, fera la différence.

14. À titre d'exemple, les écarts entre les avancées technologiques permettant de solutionner nombre de problèmes de pollution et leur mise à l'écart par les intérêts financiers plus puissants les scandalisent. Plus d'un garçon a rapporté le cas de voiture pouvant carburer à l'eau et dont le brevet a été acheté par une compagnie pétrolière pour plus d'un million de dollars. 
En somme, les garçons se construisent dans un rapport complexe au monde : l'ouverture à un monde intérieur et relationnel, fait de goûts et de talents, de sentiments, de valeurs et de rêves, et à un monde extérieur, public et politique, à la fois proche, dans son omniprésence qui interfère avec leurs perceptions, leurs choix et leurs projections, et distant par sa logique marchande qui impose des valeurs individualistes et matérialistes, menaçant leurs aspirations à une vie en accord avec leurs valeurs.

À un niveau temporel, ce monde extérieur est aussi éloigné du fait qu'on y entrera plus tard, à la majorité ou à l'entrée au cégep. Entre les deux, c'est dans cet «espace potentiel » (Winnicot, 1975) dans lequel se retrouvent le jeu, la proximité, les nouvelles expériences et les défis, que chacun, avec les autres, se découvre, se compose, fait ses choix, devient soi-même, à partir de son histoire, de ce qui l'habite et l'entoure. Loin d'être des récepteurs passifs, des éponges, de leur expérience et de leur environnement, les garçons d'aujourd'hui prennent conscience, trient et font des choix.

\section{CONSTRUCTION DE SOI CHEZ LES JEUNES HOMMES : DE L'IDENTITÉ MASCULINE À LA SUBJECTIVITÉ PLURIELLE}

Au moment où j'ai commencé à m'intéresser à l'adolescence des garçons, au début des années 2000 et tout le long de mon étude dans les années qui suivirent, une réelle préoccupation entourant l'identité masculine, la place des hommes et le devenir des garçons s'est manifestée dans plusieurs champs : la santé, l'intervention, l'éducation, le milieu universitaire, la création littéraire, le cinéma documentaire et de fiction, la presse écrite. La masculinité a été une des questions sensibles du tournant du siècle. Nul besoin de revisiter toute la démarche de prise de conscience (et de débats, voire de querelles idéologiques) qu'a suscitée cette mobilisation, pour retenir qu'elle a indéniablement ouvert un espace de légitimité pour traiter des spécificités des garçons et des hommes (dans un esprit de continuité de l'approche différenciée selon les sexes que les féministes avaient proposée en 1995). Ce phénomène social s'opère dans un mouvement d'interaction réciproque entre des changements individuels et collectifs, d'une part, et des transformations institutionnelles et sociétales, d'autre part.

En écoutant les garçons de cette étude, il m'a semblé que la notion d'identité masculine perdait peu à peu son statut de diktat parce que, simplement, elle ne résonne plus avec autant de conviction dans le vécu des adolescents.

Ils ont grandi avec la mixité et l'égalité, ce sont leurs mots, ce qui fait que sous le rapport de la socialité, ces garçons perçoivent le masculin et le féminin avec une certaine fluidité. La démarcation franche entre les humains gars et filles s'estompe. À part le physique (« un corps d'homme ou de femme »), ce qui fait la différence est, selon eux, beaucoup plus une question de personne que d'appartenance à un sexe/genre. Ils ont pour la plupart été encouragés à développer leurs talents artistiques, leur sensibilité et l'expression de leur ressenti. Ils ont observé le potentiel compétitif des filles en matière de sport, d'études et de technologie. Cette perméabilité entre les genres, par rapport aux stéréotypes, n'exclut pas les constats de différences. Par exemple, ils rapportent la pression supplémentaire qui pèse sur leurs consœurs en matière d'apparence physique et l'intensité émotive avec laquelle elles vivent leurs relations. 
Seulement, d'après les garçons de cette étude, ces différences sont plutôt périphériques, elles ne suffisent pas à fonder une division radicale entre les deux groupes.

Ce qui ne veut pas dire que la reconnaissance d'être un gars n'existe pas, mais elle se conjugue autrement et dans un spectre plus large de possibilités. L'adhésion à des codes de virilité dans les pratiques de football (la souffrance et l'oubli du corps, l'intolérance à la faiblesse, « je ne supporte pas les moumounes », etc.) semblent pouvoir cohabiter sans problème avec ceux traditionnellement associés à la féminité : la préoccupation reconnue pour leur tenue vestimentaire, leur coiffure et la shape de leur corps.

La spontanéité, la permissivité et l'aisance avec lesquelles les garçons se livrent et se racontent laissent entrevoir qu'ils se construisent (et se voient) avant tout en tant que personnes. Sans chercher à se mettre en valeur à tout prix ou à correspondre à un idéal de masculinité, une fois en confiance, les garçons parlent sans retenue aussi bien de ce qu'ils considèrent comme leurs succès et leurs fiertés que de ce qui serait leurs limites, leurs travers et leurs points sensibles. Certes, la compétition marque les rapports entre gars, mais ils en sont conscients et elle ne représente ni la seule voie possible ni nécessairement la plus édifiante. En revanche, les relations, et surtout leur qualité, deviennent un pôle important de construction de soi.

À ce chapitre, il est bon de rappeler que ces garçons ont grandi avec les séparations et les nouvelles unions de leurs parents. Ils pensent beaucoup plus leurs références significatives en fonction de la qualité des relations qu'en matière de stabilité d'une structure familiale (qui n'a pas de réelle importance, sauf pour ceux - une minorité - dont les parents sont encore ensemble). La signification que prennent les personnes, qu'elles soient de leur âge ou d'une autre génération, dépend du respect, de l'authenticité et de la confiance, présents dans la relation. La grande majorité trouve dans la pluralité de lieux et de personnes qu'ils côtoient leurs points d'appui quand ils en ont besoin. Ils ne cherchent pas des modèles de perfection (même si certains héros les inspirent ${ }^{15}$ ), ils savent reconnaître les forces, les apports significatifs de leur entourage, de leurs parents par exemple, tout en refusant certains aspects qu'ils comptent bien laisser de côté et éviter de reproduire.

Tout porte à croire que pour ces jeunes, devenir UN HOMME semble se moduler en devenir SOI. Plutôt qu'une réussite de virilité (avec ses codes, ses prescriptions et ses interdits), le devenir-soi se comprend comme un processus très personnel tout en étant soumis à des conditions de possibilité qui débordent les frontières de l'individu. En ce sens, il ne s'agit pas tant de la mise en œuvre d'étapes incorporées, prévisibles, attendues, souhaitables et définitives, qui aboutiraient au vrai homme, que d'une quête de se connaître, établir des relations, se faire une place et vivre en accord avec ses valeurs, ses rêves et ses passions.

15. Pour une analyse plus approfondie de la référence aux modèles dans la construction identitaire des garçons, voir M. Soulière (2013), « La construction identitaire des garçons : un processus en crise ou en mutation? » dans Nicole Gallant et Annie Pilote, Regard sur la construction identitaire des jeunes, Québec, Presses de l'Université Laval, p. 89-110. 
Les garçons que j'ai rencontrés expriment très bien la dimension plurielle des possibilités qui s'offrent à eux. Cette pluralité renvoie tant à l'ouverture sociale aux différentes manières de vivre et d'expérimenter leur masculinité qu'à l'ouverture personnelle à de multiples facettes de soi, qui prennent forme et se transforment dans différents contextes, avec différentes personnes, en pratiquant différentes activités.

La dimension «genrée » de la construction de soi s'imbrique dans un devenir-soi plus englobant apparaîtun processus de subjectivation continu en constante négociation avec les contextes sociaux et culturels et les enjeux de pouvoir qui les traversent - d'où cette idée de subjectivité plurielle. II incombe à chacun la responsabilité (et le devoir) de se réaliser comme personne, en préservant ses rêves, ses passions et ses valeurs dans un monde qui à la fois offre et contraint. II est question de cheminements particuliers, originaux, faits de négociation, d'adhésion et de refus, fluctuant au gré des désirs et de l'engagement personnel, des possibilités et des contingences individuelles, sociales et politiques. La subjectivité plurielle tient de cette ouverture sur l'importance accordée à l'expérience subjective, à l'incorporation d'héritages familiaux et communautaires, au mouvement et au changement des personnes et des groupes (Seidler, 2010). La reconnaissance de ce qui est, quelle que soit sa teneur de souffrance, de honte, d'espérance, de fierté, de révolte ou de défaite, et du pouvoir de transformation tant au niveau très personnel que social et politique amène à penser les multiples possibilités de construction de soi comme intimement reliées à l'expérience et aux positionnements sociopolitiques individuels et collectifs. En ce sens, la notion de subjectivité plurielle renvoie à une interconnexion entre le dehors et le dedans, entre les pratiques et les discours dominants, les parcours individuels et collectifs et le devenir du monde. Le Soi, l'Autre et le monde constituent cette pluralité indissociable, cette subjectivité plurielle qui, maintenant plus que jamais, laisse entendre que rien n'est définitivement établi et que l'espoir est toujours permis.

\section{POUR CONCLURE}

Les changements sociaux s'opèrent lorsque des individus et des groupes contestent l'ordre, les pratiques et les discours dominants en empruntant des voies nouvelles, en nommant autrement, en expérimentant des réalités inédites. Avec le temps, ces voix marginales s'intègrent aux pratiques et aux discours dominants, reconfigurent la norme qui elle-même produit de nouveaux repères, qui seront à leur tour contestés et retransformés par le cumul des transgressions, de nouvelles expérimentations et des reformulations que feront des individus et des groupes (Comaroff, 1991).

Ce que les jeunes âgés de 14 à 17 ans de cette étude ont raconté met en effet en lumière de nouvelles pistes pour penser et comprendre la construction de soi des garçons d'aujourd'hui. Ces nouvelles pistes se dessinent dans le croisement du contexte socioculturel de leur croissance $^{16}$, de leur expérience d'adolescent et de la conjoncture historique actuelle. Ils doivent agencer une liberté d'être et de devenir dans un monde de contrôle et d'incertitude. Ils se perçoivent en grande partie comme les auteurs de leur vie, des acteurs qui font des choix. Ils

16. Ne pas oublier l'appartenance de l'ensemble des répondants à la culture du groupe dominant, canadien-français, du Québec. 
se construisent à partir de ce qu'ils ont reçu et reçoivent de la société et de l'époque où ils vivent. Une société d'abondance, aux multiples possibilités qui, lorsqu'ils étaient enfants, leur a fait une grande place, les respectant comme des personnes et les encourageant à se connaître, à exprimer leurs idées et leurs émotions.

Toutefois le sentiment de se déployer, d'avoir la liberté de se construire comme personne dans une société riche se bute à un monde extérieur qu'ils jugent sévèrement et qui les dépasse. La centralité de la logique économique est à la fois parfaitement intégrée et contestée par le biais de ses effets sur les relations, l'avenir de la planète et du monde.

En dépit de ces constats, à ce moment de leur vie, au secondaire et au cœur de leur adolescence, ils se représentent comme étant dans un moment entre deux, ce qui en quelque sorte leur permet de profiter à fond du présent tout en rêvant un avenir qui, ils le savent, leur appartient.

Parce que nous autres on est plus rêveur peut-être... [...] je pense qu'à notre âge, on voit l'avenir et on s'imagine plein d'affaires et on rêve de plein d'affaires.

Loïc, 16 ans

C'est nous le futur dans le fond.

Louis, 16 ans

Marguerite Soulière, Ph. D (anthropologie) Professeure adjointe École de service social, local 12032

Faculté des sciences sociales Université d'Ottawa 


\section{BIBLIOGRAPHIE}

Arendt, Hannah (1972). La crise de la culture. Huit exercices de pensée politique, Paris, Gallimard.

Beck, U. (2001). La société du risque. Sur la voie d'une autre modernité, Paris, Flammarion.

Bohannan Paul et Mark Glazer (1988). «Lewis Henry Morgan » dans High Points in Anthropology, New York, McGraw-Hill, p. 29-60.

Bouchard. J.-F. (2011). "Piloter" une école secondaire multiethnique en milieu défavorisé et devenir une école... "phare"! » dans Au secours, aidez-moi..., mais foutez-moi la paix! Enjeux et multiples visages de l'adolescence. Colloque au Congrès de l'ACFAS, Sherbrooke, mai 2011.

Bruner, Edward W. (1986). « Experience and its Expression. » dans Victor W. Turner et Edward W. Bruner (dir.), The Anthropology of Experience, Urbana, University of Illinois Press, p. 330.

Comaroff, Jean, et John Comaroff (1991). Of Revelation and Revolution: Christianity, Colonialism and Consciousness in South Africa, Chicago, University of Chicago Press.

Cousineau, M.-M. (2011). « Des jeunes en difficulté... mais pas juste des jeunes en difficulté » dans $A u$ secours, aidez-moi..., mais foutez-moi la paix! Enjeux et multiples visages de l'adolescence. Colloque au Congrès de l'ACFAS, Sherbrooke, mai 2011.

Dagenais, Daniel (2000). La fin de la famille moderne. La signification des transformations contemporaines de la famille, Sainte-Foy, Québec, Presses de l'Université Laval.

Di Méo, G. (1999). « Géographies tranquilles du quotidien. Une analyse de la contribution des sciences sociales et de la géographie à l'étude des pratiques spatiales ». Cahiers de Géographie du Québec, vol. 43, n 118, p. 75-93.

Dubar, Claude (2000). La crise des identités. L'interprétation d'une mutation. Paris, Presses universitaires de France.

Dulac, G. (2003). « Masculinité et intimité » Sociologie et sociétés, vol. 35, n² 2, p. 9-34.

Duret, P. (2008). « Les sportifs et le processus d'autonomisation », dans David Le Breton (dir.), Cultures adolescentes. Entre turbulences et construction de soi, Paris, Autrement.

Ehrenberg, Alain (2000). La fatigue d'être soi. Dépression et société, Paris, Odile Jacob.

Galland, Olivier (2011). Sociologie de la jeunesse, Paris, Armand Colin.

Girard, Marc (2011). «Qu'est-ce qu'un adolescent normal ? » dans Au secours, aidez-moi..., mais foutez-moi la paix ! Enjeux et multiples visages de l'adolescence. Colloque au Congrès de l'ACFAS, Sherbrooke, mai 2011.

Kaufmann, Jean-Claude (2007). L'invention de soi. Une théorie de l'identité, Paris, Hachette. 
Kelly, P. (2003). « Growing Up as Risky Business? Risks, Surveillance and the Institutionalized Mistrust of Youth », Journal of Youth Studies, vol. 6, $n^{0}$ 2, p.165-180.

Kelly, P. (2007). «Governing individualized risk biographies: new class intellectuals and the problem of youth at-risk », British Journal of sociology of Education, vol. 28, n 1, p. 39-53.

Le Breton, David (2003). L'adolescence à risque, Paris, Hachette Littératures.

Le Breton, David (2007). En souffrance. Adolescence et entrée dans la vie, Paris, Métailié.

Lesko, N. (1996). «Denaturalizing Adolescence: The Politics of contemporary Representations », Youth and Society, vol. 28, $n^{\circ}$ 2, p.139-161.

Lightfoot, Cynthia (1997). The Culture of Adolescent Risk-Taking, New York, Guilford Press.

Lock, M. (1993). «Cultivating the Body: Anthropology and Epistemologies of Bodily Practices and Knowledge », Annual Review of Anthropology, vol. 2, p. 133-155.

Niesyto, H. (2000). « Youth Research on Video Self-productions. Reflections on a Socialaesthetic Approach », Visual Sociology, vol. 15, p. 135-153.

Passerini, L. (1994). «La jeunesse comme métaphore du changement social » dans Giovanni Levi et Jean-Claude Schmitt (dir.), Histoire des jeunes en Occident. L'époque contemporaine, Paris, Seuil, vol. 2, p. 339-408.

Peretti-Watel, Patrick (2000). Sociologie du risqué, Paris, Armand Colin.

Petrella, Riccardo (2007). Pour une nouvelle narration du monde, Montréal, Écosociété.

Piron, F. (1992). «Les enjeux de la production des connaissances: essai sur le pouvoir, le savoir et la solidarité féministe », Culture, vol. 12, n² 2, p. 63-82.

Scheper-Hughes, N. et M. Lock. (1987). « The Mindful Body: A Prolegomenom to Future Work in Medical Anthropology », Medical Anthropology Quarterly, vol. 1, p. 6-41.

Seidler, V.J. (2010). Embodying Identities: Culture,Differences ansd Social Theory, Bristol et Portland, Policy Press.

Singly, François de (2006). Les adonaissants. Paris, Armand Colin.

Soulière, Marguerite (2013). «La construction identitaire des garçons : un processus en crise ou en mutation? » dans N. Gallant et A. Pilote Regard sur la construction identitaire des jeunes, Québec, Presses de l'Université Laval, p.89-110.

Vadeboncoeur, Jennifer A. (2005). « Naturalised, Restricted, Packaged, and Sold: Reifying the Fictions of "Adolescent" and "Adolescence" 》 dans Lisa Patel Stevens et Jennifer $A$. Vadeboncoeur Re/constructing "the adolescent": Sign, Symbol, and Body, New York, Peter Lang, p.1-24.

Winnicott, Donald Woods (1975). Jeu et réalité. L'espace potentiel. Paris, Gallimard Jeunesse. 